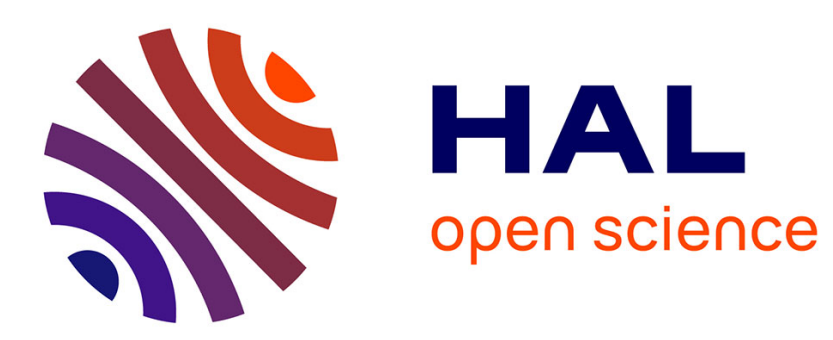

\title{
A multifactorial investigation of captive chimpanzees' intraspecific gestural laterality
}

Jacques Prieur, Simone Pika, Stéphanie Barbu, Catherine Blois-Heulin

\section{To cite this version:}

Jacques Prieur, Simone Pika, Stéphanie Barbu, Catherine Blois-Heulin. A multifactorial investigation of captive chimpanzees' intraspecific gestural laterality. Animal Behaviour, 2016, 116, pp.31-43. 10.1016/j.anbehav.2016.03.024 . hal-01308008

\section{HAL Id: hal-01308008 \\ https://hal-univ-rennes1.archives-ouvertes.fr/hal-01308008}

Submitted on 7 Jul 2016

HAL is a multi-disciplinary open access archive for the deposit and dissemination of scientific research documents, whether they are published or not. The documents may come from teaching and research institutions in France or abroad, or from public or private research centers.
L'archive ouverte pluridisciplinaire HAL, est destinée au dépôt et à la diffusion de documents scientifiques de niveau recherche, publiés ou non, émanant des établissements d'enseignement et de recherche français ou étrangers, des laboratoires publics ou privés. 


\section{A multifactorial investigation of captive chimpanzees' intraspecific gestural laterality}

Jacques Prieur ${ }^{\mathrm{a}}$, Simone Pika ${ }^{\mathrm{b}}$, Stéphanie Barbu ${ }^{\mathrm{a}}$, Catherine Blois-Heulin ${ }^{\mathrm{a}}$.

${ }^{\text {a }}$ Ethos 'Ethologie Animale et Humaine', Université de Rennes 1-CNRS UMR 6552, Station biologique de Paimpont, France.

${ }^{\mathrm{b}}$ Max Planck Institute for Ornithology, Humboldt Research Group 'Comparative Gestural Signalling', Seewiesen, Germany

Article history:

Received 19 May 2015

Initial acceptance 7 July 2015

Final acceptance 2 March 2016

MS. number: 15-00425R

Correspondence: J. Prieur, UMR 6552, Station Biologique, Université de Rennes 1-CNRS, 35380 Paimpont, France.

E-mail: jac.prieur@yahoo.fr 
Social laterality is the core of two major theories: one concerns the evolution of laterality at the population level and the other the evolution of human language. However, few studies have investigated gestural laterality in communication between conspecifics. To our knowledge, the present study is the first to investigate the production of intraspecific gestures taking into account the influence of multiple factors on gestural laterality: first, gestural characteristics (sensory modality, use of a communication tool, sharing degree in the population and duration); second, the interactional context (visual field and body sides of signaller and recipient, and emotional context); and third, individual sociodemographic characteristics of signaller and recipient (age, sex, group, hierarchy, affiliation and kinship). We questioned, first, whether gestural laterality differed with gesture at the population level and second, whether some factors influenced gestural laterality. To do so, we evaluated social laterality in dyadic interactions in 39 chimpanzees, Pan troglodytes, living in three groups in captivity. We found that, at the population level, 13 of the 21 gestures we observed were performed predominantly with the right hand. Gestural laterality of signallers was influenced mainly by interactional context, gesture characteristics (except gesture duration) and signallers' hierarchical rank and age. Signallers used their hand ipsilateral to recipients for tactile and visual gestures and their contralateral hand for gestures involving auditory communication and a communication tool. Moreover, signallers' use of their right hand was more important for subordinates. This was also true in negative contexts for gestures common to most of the subjects. Our results further support the hypothesis that laterality in gestural communication might represent a precursor of the left-hemispheric lateralization of language. We discuss our results in relation to theories concerning the origins of cerebral hemispheric lateralization and their consistency with previous studies.

Keywords: brain asymmetry, chimpanzee, communication, gesture, social laterality. 
Laterality in social behaviour is becoming an important research area as it is the core of two major theories: the first concerns the evolution of laterality at the population level (ELP) and the second the origin of human language (OHL). The ELP theory (e.g. Ghirlanda \& Vallortigara 2004; Vallortigara \& Rogers 2005; Ghirlanda et al. 2009) hypothesizes that the evolution of population level asymmetries is influenced by social behaviour. It suggests that behavioural laterality at the population level emerged in species subject to selection pressures imposed by social interactions rather than in solitary species. The gestural OHL theory (e.g. Arbib et al. 2008; Corballis 2002; McNeill 2012) hypothesizes an evolutionary relationship between the roots of human language and handedness. It postulates that the left-cerebral lateralization of language evolved from gestural communication.

According to the ELP theory, brain lateralization may have evolved in two steps. First, biases at the individual level would have been selected because they increase brain efficiency (e.g. see Rogers et al. 2004 for review). Second, biases at the population level could have emerged from an evolutionarily stable strategy (ESS)/frequency-dependent selection based on interspecific prey-predator interactions. More recently, Ghirlanda and colleagues (2009) proposed that the pattern of population level laterality could be better explained by an ESS based on a trade-off between competitive and cooperative intraspecific interactions than by interspecific interactions. Social laterality could have appeared at the population level through social pressures (e.g. Vallortigara \& Rogers 2005) and because it facilitated intraspecific interactions (Rogers 2000). This view is supported by empirical data on fish (e.g. Bisazza et al. 2000) and tadpoles (e.g. Bisazza et al. 2002) showing that population level laterality is more likely to be exhibited by social than solitary species.

Among laterality expressed in social interactions, laterality of gestural communication of our closest living relatives, the great apes, is the focus of an ever-growing body of research (e.g. see Hopkins et al. 2012 for review) participating in the perennially vivid scientific 
debates on the origins of language by providing recent arguments in favour of a gestural origin. Below, we refer to gestures as 'movements of the limbs or head and body directed towards a recipient that are goal-directed, mechanically ineffective (that is, they are not designed to act as direct physical agents) and receive a voluntary response' (Pika \& Bugnyar 2011, p 4). All the properties underlying the production and use of sophisticated gestural communication (e.g. intentionality and flexibility) are crucial prerequisites for human language (e.g. see Hopkins et al. 2012; Meguerditchian et al. 2013 for reviews). However, despite the challenges, intraspecific data remain sparse in the literature (Forrester et al. 2012), and this is all the more the case for gestures for which laterality has been more extensively investigated, in captive conditions, in communication directed towards humans. Therefore, whether most frequent spontaneous gestures directed towards conspecifics are lateralized at the population level remains open.

In addition, many factors have been found to modulate laterality expressed in gestural communication (e.g. gesture type, relative positions of subjects during an interaction, emotional valence and sociodemographic components). For instance, Hobaiter and Byrne (2013) showed that chimpanzees, Pan troglodytes, in the wild use their right hands significantly more for object manipulation gestures than for nonobject manipulation gestures. Moreover, captive chimpanzees used their right hands more for begging to humans than for pointing at them (Hopkins \& Leavens 1998; Hopkins \& Wesley 2002). Therefore, the type of chimpanzees' gestures seems to have a crucial impact on the direction and strength of hand use during communication. This factor could explain discrepancies within studies focusing on different gestures (e.g. Hopkins \& Leavens 1998; Hopkins \& Wesley 2002). Investigating the effect of gesture type on laterality requires considering various elementary gestural characteristics (e.g. sensory modality: tactile, visual and auditory; with or without use of a communication tool; degree of sharing among the population: rare or common; duration: short 
or long). However, which factors could explain why observational studies of behavioural laterality focusing on the same gesture have provided different results (e.g. Fletcher's (2006) and Meguerditchian and colleagues' (2012) studies of chimpanzees for Clap hand directed towards humans)?

Taking into account multiple elementary factors related to social interactions (e.g. interactional context components and sociodemographic factors) should help us to explain heterogeneous results between studies. This multifactorial approach could also be particularly relevant to investigate possible effects of social pressures on laterality. Surprisingly, relatively little is known about the impact of the position of the recipient (most often a human) on primates' hand preference. To date, authors have reported the influence of the experimenter's position on hand preference for Food beg and Pointing (pooled data) by chimpanzees (Hopkins \& Wesley 2002), but not for Food beg by olive baboons, Papio anubis (Bourjade et al. 2013). Concerning the emotional valence of the context, intraspecific agonistic interactions generally induce a preferential use of the left visual field by many vertebrates (e.g. gelada baboons, Theropithecus gelada: Casperd \& Dunbar 1996). In contrast, Chapelain and colleagues (n.d.) found a left visual field bias for bonobos, Pan paniscus, during positive interactions. These studies highlight complex interactions between the respective positions of signaller and recipient (for both body side and visual field) and the emotional context, interactions that require further investigations to understand better their influence on primates' gestural communication with conspecifics.

Concerning sociodemographic factors that may be particularly associated with social pressure acting on laterality, a few studies have investigated the effect of age on gestural laterality. For example, chimpanzees' right direction in hand preference increased with age in the wild (Hobaiter \& Byrne 2013) and in captive environments (Hopkins \& Leavens 1998). However, age effects have not been consistently found across studies (e.g. chimpanzees: 
Hopkins et al. 2005a). Regarding a sex effect, as far as we know, only two studies have reported such an effect, but with opposite results: Hopkins and Leavens (1998) found that male chimpanzees tended to be less right-handed than females, whereas Hopkins and de Waal (1995) found that male bonobos were more right-handed than females. Other studies did not find an influence of sex on nonhuman primates' laterality in gestures (e.g. chimpanzees: Hopkins et al. 2005a). Studying a group effect can also help us shed light on possible effects of social pressures on gestural laterality. To date, an absence of a group effect on gestural laterality has been found for captive chimpanzees for human-directed Clapping (Meguerditchian et al. 2012) and for Throwing directed towards both humans and conspecifics (pooled data; Hopkins et al. 2005b) as well as for captive olive baboons for Hand slap directed towards both humans and conspecifics (pooled data; Meguerditchian et al. 2011). Concerning social factors, to our knowledge, only one study has investigated a kinship effect: Hopkins and colleagues' (2005b) study of captive chimpanzees' hand preference for Throwing did not show an influence of kinship. Hierarchical rank effects have been investigated only in visual laterality of two species of mangabeys, Cercocebus torquatus torquatus and Lophocebus albigena albigena: Baraud and colleagues (2009) showed that high-ranking mangabeys were approached more often from their left than from their right. Although the quality of the relationship (i.e. affiliation) may also represent a possible source of social pressure acting on laterality, the influence of affiliation remains undocumented so far. Therefore, the full range of individual sociodemographic characteristics remains to be taken into consideration simultaneously to assess as rigorously as possible their relative weights and possible influences on primates' gestural communication.

To date, most studies have focused on some particular factors in isolation providing a fragmented picture of the issue and contradictory results. This emphasizes the importance of investigating further nonhuman primates' gestural communication to improve our 
understanding of the origin and evolution of both social laterality at a population level and of language. To our knowledge, no previous study has assessed gestural laterality using a comprehensive approach simultaneously taking into account multiple influential factors and their interactions as well as considering sociodemographic characteristics and narrow categories of age (e.g. immature, adolescent, young and mature adult and elder) and hierarchy (e.g. dominant, intermediate and subordinate) of both signaller and recipient, essential requirements to avoid biases and to yield unambiguous results. Although socioecologically relevant conditions close to conditions in which natural selection has acted are of particular interest to study gestural laterality in an evolutionary perspective, many studies have investigated nonhuman primates' gestural communication in artificial situations with human experimenters (e.g. see Meguerditchian et al. 2013 for a review). Only a few studies have analysed gestural laterality during spontaneous communication strictly between conspecifics (chimpanzees: Fletcher \& Weghorst 2005; Meguerditchian et al. 2010; Hobaiter \& Byrne 2013; bonobos: Chapelain 2010; olive baboons: Meguerditchian \& Vauclair 2006).

The main aim of this study was to understand better intraspecific gestural laterality and the factors influencing hand preferences in one of humans' closest living relatives, chimpanzees. In particular, we wondered (1) whether it is possible to find an effect of social pressures on intraspecific communication considering multiple factors related to social interactions and (2) whether some gesture characteristics are better markers than others of the righthandedness/left-brain specialization for language. To this end, we investigated systematically the production of the most frequent gesture types of their communication repertoire (e.g. Nishida et al. 2010). We designed and applied a methodology to assess and to compare as unambiguously as possible the respective influences of factors expected to modulate laterality in gestural communication. Our first question was: is there a gestural laterality bias at the population level (in this study our population includes all our subjects)? To answer this 
question, we analysed each of 21 gestures separately. As seen in the human literature (e.g. Kimura 1973), we predicted that a majority of these frequently expressed gestures would be right-lateralized at the population level. Our second question was: which factors influence gestural laterality? To answer this question, the three following categories of factors were taken into account simultaneously: the interactional context components (visual field and body side of both signaller and recipient and the emotional valence of the context), gesture characteristics (sensory modality, use of a communication tool, degree of sharing among the population and duration) and individual sociodemographic characteristics of both signaller and recipient (age, sex, group, hierarchy, kinship and affiliation). Based on the reports mentioned above, we predicted that signallers' gestural laterality would be particularly modulated by interactional context as well as gesture and individual social characteristics. Furthermore, we expected to find an effect of social pressures on gestural laterality particularly through the influence of individual social characteristics (e.g. signaller's hierarchical status).

\section{Methods}

\section{Subjects}

Thirty-nine chimpanzees raised under seminatural conditions were observed in three zoos: Leipzig Zoo, Germany $(N=16)$, Beauval Zoo $(N=14)$ and La Palmyre Zoo $(N=9)$ (France). Following Goodall (1986), age categories of the subjects were defined as follows: immatures (0-7 years old), adolescents (8-12 years old), young adults (13-20 years old), mature adults (21-35 years old) and elderly (over 35 years old). Our population of chimpanzees (26 females and 13 males) comprised eight immatures, six adolescents, seven young adults, 11 mature 
adults and seven elders. All outdoor enclosures offer seminatural environment surrounded by a water ditch and contained climbing structures (e.g. trees, ropes and platforms) as well as vegetation (e.g. bamboos and various types of bushes and grass). All indoor enclosures also included climbing structures. Zookeepers fed the study subjects with diverse types of food and enrichments. Water was available ad libitum.

\section{Ethical Note}

As the study was noninvasive and involved only observations of animals in their enclosures, neither experimental permits nor ethical approvals were required.

\section{Observation procedures}

Observation data were collected in 2013 at the Wolfgang Köhler Primate Research Center at the Leipzig Zoo (1 July-20 September), at Beauval Zoo (29 September-10 November) and at La Palmyre Zoo (23 November-22 December). This yielded, respectively, $333 \mathrm{~h}, 198 \mathrm{~h}$ and $174 \mathrm{~h}$ of observations, for a total of $705 \mathrm{~h}$.

During observation days, data were collected during four $1.5 \mathrm{~h}$ sessions, two in the morning and two in the afternoon. The sampling rule was 'sampling all occurrences of some behaviours' (Altmann 1974). Data were collected in real time by using a stopwatch, a powerful pair of binoculars, and a paper sheet onto which observation data were recorded. Data collection was mostly performed from above and as close as possible to the subjects. Data were only recorded when a clear view of the subjects was possible. 


\section{Coding procedure}

Only dyadic interactions were taken into account. We defined the subject that started the social interaction as the signaller and the target of this interaction as the recipient. For each dyadic interaction, we recorded (1) the type of gesture (Table 1, see below for further details) and the left or right limb (hand or foot) used by the signaller to communicate, (2) the interactional context of gestural production considering the relative positions of the two subjects before and during an interaction (both visual field and body side) as well as the emotional context associated with the interaction, and (3) the identity and role (signaller or recipient) of both subjects, as described below.

Following Pika and Bugnyar's (2011) definition of gesture, we considered only intentionally produced gestures that (1) were used to initiate (not continue) a social interaction, (2) were mechanically ineffective (Pollick \& de Waal 2007) and (3) included gazing at the recipient, gaze alternation and/or waiting for a response (e.g. Tomasello et al. 1989).

Because only two gestures involved the foot (Slap foot and Kick), we used the term 'hand' instead of 'limb' for simplicity. The hand used to communicate was recorded during dyadic interactions only when both hands of the signaller were free and symmetrically positioned with respect to the subject's body midline before the interaction, without any environmental factors that could influence the use of one hand (e.g. close to a wall/bush/tree).

Data were recorded when a gesture was produced either singly or in a gesture bout (i.e. sequence of gestures separated by intervals; e.g. Marchant \& McGrew 1991). Only the first gesture of a bout was recorded. The following criteria had to be met to consider that a single gesture or a bout was terminated: (1) the signaller's hand returned to its initial position (Meguerditchian et al. 2010), (2) the signaller switched to another noncommunication activity (e.g. forage) or (3) the movement was influenced by an outside incident (e.g. stumble; e.g. 
Harisson \& Nystrom 2010). A change in hand activity had to last more than $3 \mathrm{~s}$ before another gesture could be taken into account thus ensuring statistical independence of data (e.g. Hopkins \& de Waal 1995).

\section{Gesture characteristics}

Our gesture classification was based on previous gestural repertoires (when necessary anatomical elements or precisions were added; Tables 1 and 2). Twenty-one different gestures were considered. In accordance with Pika and colleagues (2003), these gestures were divided into three communication modalities: visual gestures $(N=7)$, auditory gestures $(N=3)$ or tactile gestures $(N=11)$. These gestures were performed either with $(N=5)$ or without $(N=16)$ an object used as a communication tool. We measured the time subjects took to perform a single gesture: the starting point was determined by a hand starting to move, the end point when the hand was again in a resting position (e.g. McNeill 1992). Gestures lasting less than 2 s were categorized as 'short' gestures $(N=12)$ and gestures lasting more than $2 \mathrm{~s}$ were categorized as 'long' gestures $(N=9)$. Gestures were also divided as follows: eight of the 21 gestures, each performed by fewer than 14 subjects, were categorized as 'rare' gestures, defined as gestures performed by only a few subjects in our population (represented by our 39 subjects) and the 13 other gestures performed by at least 25 subjects were categorized as 'common' gestures, defined as gestures performed by most of the subjects in the population.

\section{Characteristics of the interactional context of gesture production}

For each dyadic interaction, we recorded the relative positions (visual field used and exposed body side) of both subjects before (the last position for $2 \mathrm{~s}$ before an interaction) and during the interaction. Most interactions were predictable as signallers produced intentional signals (e.g. gazing at the recipient, gaze alternation, movement towards the recipient). As 
detailed in Prieur (2015), because of strong positive correlations between recorded position variables, we only retained the two following position variables in the present study: the position of the recipient in the signaller's visual field during interaction (noted SVF) and the position of the signaller in the recipient's visual field during interaction (noted RVF).

The emotional contexts of interactions were divided into two categories, positive and negative, according to three criteria. The emotional context was inferred primarily according to (1) the functional consequences of the gesture during an interaction (the response of the recipient to the signaller's gesture), but also, if necessary, (2) the global social context in which the given interaction occurred and (3) the signaller's facial (e.g. Parr \& Waller 2006) and vocal (Crockford \& Boesch 2005) expressions and, to a lesser extent, whole-body expressions (e.g. rhythmic movements: Goodall 1989; piloerection: Van Hooff 1973).

\section{Sociodemographic characteristics of the subjects}

In addition to individual demographic characteristics, such as age and sex, we considered data concerning kin and social relationships (affiliation and hierarchy).

\section{Kinship}

Kinship was determined by genetic analyses and data were provided by each zoo. Three categories of chimpanzee pairs were considered: (1) 'Parent-infant' including mother-infant and father-infant pairs, (2) 'Siblings' including siblings and half-siblings and (3) 'Unrelated' for pairs of genetically unrelated subjects. 


\section{Affiliation}

In accordance with Pollick and de Waal's (2007) definition of affiliative and agonistic behaviours we selected the following six strict affiliative gestures (gestures that are expressed only in positive contexts; 8986 interactions in total) to quantify affiliation: Embrace, Embrace half, Embrace lateral, Embrace ventral/dorsal, Extend hand and Touch body. We analysed all agonistic interactions recorded (4334).

Two indexes of interest have already been used to evaluate relationship quality (Weaver \& de Waal 2002; Silk et al. 2013). To remedy disadvantages of these two indexes and to better evaluate relationship quality within pairs of individuals (Prieur 2015), we created a dyadic affiliation index (DAI) to assess relationship quality based on the relative frequencies of affiliative and agonistic behaviours within the dyad. This index increases with affinity, starting from 0 in the absence of affinity. It is calculated as:

$$
\mathrm{DAI}_{x y}=\frac{\left(\frac{\sum_{i=1}^{n} \frac{f_{i x y}}{\bar{f}_{i}}}{n}\right)}{1+\left(\frac{\sum_{j=1}^{n^{\prime}} \mathrm{h}_{j x y}}{\overline{\mathrm{h}}_{j}}\right)}
$$

where $f_{i x y}$ is the total number of affiliative interactions of the behaviour (i) expressed by $x$ towards $y ; \bar{f}_{i}$ is the mean number of affiliative interactions of the behaviour $(i)$ across all dyads; $n$ is the number of affiliative behaviours expressed by $x$ towards $y ; h_{j x y}$ is the total number of agonistic interactions of the behaviour $(j)$ expressed by $x$ towards $y ; \bar{h}_{j}$ is the mean number of agonistic interactions of the behaviour $(j)$ across all dyads; $n$ ' is the number of agonistic behaviours expressed by $x$ towards $y$. Three categories of dyadic affiliation were considered: (1) 'Low' from 0 to 0.5 (389 dyads), (2) 'Medium' from 0.5 to 1 (58 dyads) and (3) 'High' more than 1 (47 dyads). 


\section{Hierarchy}

Following Langbein and Puppe (2004), hierarchical dominance relationships were determined on the basis of agonistic interactions (Pollick \& de Waal 2007). Only interactions within dyads for which the aggressor and the receiver of the threat were clearly identified were taken into account. All recorded agonistic interactions (4334) were considered. We organized these interactions into sociometric matrices. The dominance hierarchies were established using MatMan 1.1 (Noldus Information Technology, Wageningen, Netherlands; de Vries 1995, 1998; de Vries et al. 2006). Each of the $N$ subjects in one zoo was assigned a rank from 1 (the most dominant) to $N$ (the most subordinate). Three categories of hierarchical rank were considered: 'Subordinate', 'Intermediate' and 'Dominant' (Beauval group: five subordinates, five intermediates and four dominants; Leipzig group: five subordinates, five intermediates and six dominants; Palmyre group: three subordinates, three intermediates and three dominants).

Statistical analysis

All statistical analyses were conducted with R version 3.0.3 (R Development Core Team 2014). The level of significance was set at 0.05 .

\section{Descriptive Statistics}

To enable subsequent statistical analyses (binomial test), we included data only for gestures that had been recorded at least six times each by at least six subjects (Chapelain 2010). Binomial tests on the numbers of responses involving the left and right hands assessed individual level biases for each gesture. A subject exhibiting a significant bias (respectively no bias) was categorized as lateralized (respectively nonlateralized). Direction of gestural 
asymmetry was evaluated by calculating an individual handedness index (HI) for each subject applying the formula $\mathrm{HI}=(\mathrm{R}-\mathrm{L}) /(\mathrm{R}+\mathrm{L})$, where $\mathrm{R}$ and $\mathrm{L}$ represent the total number of rightand left-hand responses, respectively. HI varies from -1.0 to +1.0 . Its sign indicates direction of hand preference: positive values correspond to a right-hand preference and negative values to a left-hand preference. The strength of individual hand preference was estimated by the absolute value of HI (ABSHI). This procedure is similar to that used by previous authors (e.g. Harris \& Carlson 1993).

Binomial tests assessed population level biases in the number of lateralized and nonlateralized subjects for each gesture. When at least six subjects were lateralized, binomial tests assessed population level biases in the number of right-handers and left-handers for each gesture. Considering laterality on a continuum (e.g. McGrew \& Marchant 1997) rather than dichotomously, we evaluated the bias in hand use at the population level by a one-sample two-tailed Student's t test on the HI values of all subjects only when the HI distribution was normal (Shapiro-Wilk normality test) and by a one-sample Wilcoxon signed-rank test when the HI distribution was not normal. Pearson rank correlation tests checked possible correlations between the visual field and body side of both signaller and recipient as well as before and during an interaction.

\section{Generalized linear mixed model analysis on the multiple influential factors}

We evaluated the possible effect of multiple variables on gestural laterality using a generalized linear mixed model (GLMM) for binary data (logistic regression) with hand use as the dependent variable. This GLMM analysis allowed estimation of the effects of interactional context as well as gesture and individual sociodemographic characteristics on hand use (see Table 3 for a descriptive summary of dependent, fixed and random variables). We included all possible interactions between fixed variables. To avoid pseudoreplication 
caused by repeated observations (Waller et al. 2013), we considered signallers' and recipients' identities as the random variables.

For the GLMM analysis, we used the 'glmer' function ('Ime4' package, Bates et al. 2014) and we selected the best model as the one with the lowest Akaike's information criterion (AIC). We visually checked equivariance, independence and normality of model residuals using the 'plotresid' function ('RVAideMemoire' package, Hervé 2014). The main effects of the best model were tested with type II Wald chi-square tests using the 'Anova' function ('car' package, Fox \& Weisberg 2011). Least square means (LSmeans) and associated adjusted probabilities of right-hand use were computed using the 'lsmeans' function ('lsmeans' package, Lenth 2014). Post hoc multiple comparisons tests were performed using Tukey's honest significant difference (HSD) test (below, referred to as 'Tukey test') and calculated between LSmeans ('Ismeans' package).

\section{Results}

We recorded 25534 gesture occurrences. After having applied the statistical criteria required for performing the binomial test (Siegel \& Castellan 1988), 25024 gesture occurrences were retained for descriptive statistics and related analyses. The mean number of gesture occurrences per subject was 641.64 (minimum=29, maximum=3 198; $\mathrm{SD}=764.16$ ).

\section{Gestural laterality at the population level}

To estimate gestural laterality at the population level, we analysed each of the 21 gestures separately. Significantly more subjects were nonlateralized than lateralized for eight tactile and two visual gestures (binomial test: tactile gestures: $P \leq 0.008$; visual gestures: $P \leq 0.023$; 
Table 2), the average percentage of nonlateralized subjects for all gestures was $66.86 \%$ (minimum=12.5, maximum $=100 ; \mathrm{SD}=22.47$. Analyses revealed that significantly more subjects were right-handed than left-handed for the following six gestures (binomial test: $P \leq$ 0.001; Table 2): two auditory (Slap hand and Slap foot), one tactile (Punch) and three visual gestures (Shake object, Extend hand and Raise arm).

Considering laterality on a continuum, rather than dichotomously, we found a significant right-hand bias at the population level for 13 gestures (one-sample two-tailed t test or onesample Wilcoxon signed-rank test: $P \leq 0.024$; Table 2). The average Mean HI was 0.21 (minimum=-0.15, maximum $=0.47 ; \mathrm{SD}=0.19$ ) and the average Mean ABSHI was 0.36 (minimum $=0.15$, maximum $=0.84 ; \mathrm{SD}=0.16$ ) for all gestures.

Factors and their mutual interactions influencing gestural laterality

To investigate factors influencing gestural laterality, we focused on interactional context components, gesture characteristics and individual sociodemographic characteristics. We carried out a GLMM analysis taking into account all the 25534 gesture occurrences. The mean number of gesture occurrences per subject associated was 654.72 (minimum=47, maximum=3 199; $\mathrm{SD}=758.80)$.

Here we present a selection of results corresponding to variables for which an influence on right-hand use was found. Tables 4, 5, 6 and 7 present a summary of these selected results. For clarity, only significant $P$ values of post hoc multiple comparisons tests are given in the text below (see more details in Prieur 2015). 


\section{Interactional context}

Position of recipient in signaller's visual field during an interaction (SVF). Signallers used their right hand more when the recipient was in their right visual field (SVF_R) than in their left visual field (SVF_L) during an interaction for tactile and visual gestures as well as gestures without an object (Tukey test: for each of these variable modalities: $P<0.0001$ ). In contrast, signallers used their right hand more when the recipient was in their left visual field (in SVF_L condition) than in their right visual field (SVF_R) for auditory gestures (Tukey test: $P<0.0001)$.

Position of signaller in recipient's visual field during an interaction $(R V F)$. Signallers used their right hand more when they were in the recipient's left visual field (RVF_L) than in their right visual field (RVF_R) during an interaction for tactile and auditory gestures, gestures with and without an object as well as short and long gestures (Tukey test: tactile: $P<0.0001$; auditory: $P<0.0001$; with object: $P=0.009$; without object: $P<0.0001$; short: $P=0.015$; long: $P<0.0001)$. This was also true for parent-infant and unrelated pairs, the three youngest signaller age classes (immatures, adolescents and young adults) and whatever the signaller's hierarchical rank and the zoo (Tukey test: parent-infant and unrelated pairs: $P<0.0001$; immature: $P=0.003$; adolescent: $P<0.0001$; young adult: $P<0.0001$; dominant: $P<0.0001$; intermediate: $P=0.025$; subordinate: $P<0.0001$; Beauval, Leipzig and La Palmyre: $P<$ 0.0001).

Emotional context. Signallers were more right-handed in negative than in positive contexts when performing common gestures (Tukey test: $P=0.018$ ). 


\section{Gesture characteristics}

Use of communication tools in gestures. Signallers used their right hand more for gestures without an object than for gestures with an object when the recipient was in their right visual field (SVF_R; Tukey test: SVF_R: $P<0.0001)$. In contrast, for SVF_L signallers used their right hand more for gestures with than without an object (Tukey test: $P<0.0001$ ).

Gesture sharing degree. Signallers used their right hand more for common than for rare gestures in negative emotional contexts as well as when they were dominant or immature, for auditory gestures, for gestures directed towards a strong affiliative partner and for the Palmyre group (Tukey test: negative emotion: $P=0.021$; auditory: $P<0.0001$; dominant signaller: $P=$ 0.025; immature signaller: $P<0.0001$; strong affiliative partner: $P=0.043$; Palmyre: $P=$ 0.006). In contrast, signallers used their right hand more for rare than for common tactile gestures (Tukey test: $P=0.042$ ).

\section{Individual social characteristics}

Signaller's hierarchical rank. Subordinate signallers used their right hand more than intermediate signallers when the recipient was in their left visual field (SVF_L) as well as when the signaller was in the recipient's left visual field (RVF_L), for tactile gestures, rare gestures and gestures directed towards a medium affiliative partner (Tukey test: SVF_L: $P=$ 0.020; RVF_L: $P=0.025$; tactile: $P=0.021$, rare: $P=0.017$; medium affiliative partner: $P=$ 0.026). Furthermore, subordinate signallers used their right hand more than dominant signallers in SVF_R situation, as well as for rare gestures and when performing gestures towards a medium affiliative partner (Tukey test: SVF_R: $P=0.023$; rare: $P=0.040$; medium affiliative partner: $P=0.011)$. 
Affiliation. Subordinate signallers were less right-handed when performing gestures towards a strong than towards a medium affiliative subordinate partner (Tukey test: $P=0.018$ ).

\section{Individual demographic characteristics}

Signaller's age class. Elderly signallers were less right-handed than mature adult signallers for rare gestures, as well as whatever their location was in the recipient's visual field during the interaction (RVF), gesture sensory modality, use of a communication tool or not and affiliation of the recipient (Tukey test: rare: $P<0.0001$; RVF_R: $P<0.0001$; RVF_L: $P<$ 0.0001; tactile: $P<0.0001$; auditory: $P=0.010$; visual: $P=0.002$; without object: $P=0.010$; with object: $P<0.0001$; strong affiliative partner: $P=0.001$; medium affiliative: $P<0.0001$; low affiliative: $P=0.0004)$. They were less right-handed than young adult signallers for tactile gestures and gestures with an object, gestures directed towards medium and low affiliative partners, whatever the RVF situation was and the degree of gesture sharing (Tukey test: tactile: $P<0.0001$; with object: $P<0.0001$; medium affiliative partner: $P=0.002$; low affiliative: $P=0.005$; RVF_R: $P=0.024$; RVF_L: $P<0.0001$; rare: $P=0.004$; common: $P=$ 0.009). Elderly signallers were also less right-handed than adolescent signallers for tactile and auditory gestures, gestures with an object, gestures directed towards medium and strong affiliative partners, whatever the RVF was and the degree of gesture sharing (Tukey test: tactile: $P<0.0001$; auditory: $P=0.006$; with object: $P<0.0001$; strong affiliative: $P=0.034$; medium affiliative partner: $P=0.0002$; RVF_R: $P=0.042$; RVF_L: $P<0.0001$; rare: $P=$ 0.013; common: $P=0.001)$. They were also less right-handed than immature signallers for gestures with an object (Tukey test: $P=0.036$ ). Mature adult signallers were more righthanded than adolescent signallers in $R V F \_R$ as well as for rare gestures (Tukey test: RVF_R: $P=0.037$; rare: $P=0.004)$. They were also more right-handed than immature signallers for tactile gestures as well as for rare gestures, gestures directed towards medium and low 
affiliative partners and whatever the RVF situation was and the use of an object or not (Tukey test: tactile: $P=0.001$; rare: $P<0.0001$; medium affiliative partner: $P=0.004$; low affiliative: $P=0.029$; RVF_R: $P=0.004$; RVF_L: $P=0.035$; without object: $P=0.025$; with object: $P=$ 0.010). No statistical differences in right-hand use were found between either immature and adolescent signallers or young and mature adults.

Signaller's group (zoo). Signallers at Leipzig zoo were less right-handed than Beauval zoo's signallers for auditory gestures (Tukey test: $P=0.015$ ).

\section{Discussion}

The main aim of this study was to yield a better understanding of chimpanzees' gestural laterality by systematically evaluating the production of the most frequent gesture types of their natural repertoire. We investigated two research questions. First, is there a gestural laterality bias at the population level? Second, which factors influence gestural laterality?

First, considering laterality on a continuum, 13 of the 21 gestures considered presented a right-hand bias at the population level. Second, results of a GLMM analysis found that signallers' gestural laterality was particularly influenced by characteristics of the interaction (visual fields of both signaller and recipient, emotional context), of the gestures (sensory modality, use of a communication tool, sharing degree), and signaller's hierarchical rank and age. More precisely, signallers used their hand ipsilateral to the recipient for tactile and visual gestures and their contralateral hand for gestures involving auditory sensory modality and a communication tool. Signallers' right-hand use was particularly pronounced for subordinates. This was also true in negative contexts for common gestures. Furthermore, elderly signallers were less right-handed than all the younger age classes. 


\section{Gestural laterality at the population level}

Our findings support previous studies reporting a right-hand bias at the population level for both inter- and intraspecific communication for chimpanzees and olive baboons (e.g. see Hopkins et al. 2012; Meguerditchian et al. 2013 for reviews) indicating that laterality in gestural communication would be predominantly associated with the left hemisphere in these two species of nonhuman primates as in humans (e.g. see Cochet \& Byrne 2013 for review). Only a few studies have investigated gestural laterality in purely intraspecific communication (chimpanzees: Fletcher \& Weghorst 2005; Meguerditchian et al. 2010; Hobaiter \& Byrne 2013; bonobos: Chapelain 2010; olive baboons: Meguerditchian \& Vauclair 2006). A predominance of right-hand use was found by Meguerditchian and colleagues (2010) for 46 captive chimpanzees for a category of species-typical gestures (1241 data points) combining Threat, Extend arm and Hand slap and by Hobaiter and Byrne (2013) for wild chimpanzees (after pooling data across 54 subjects because of a relatively small number of data points) for a category of object manipulation gestures combining Object shake and Object move. This predominance was also found by Meguerditchian and Vauclair (2006) for 27 olive baboons for Hand slap (442 data points from 92 social interactions).

As most studies on laterality have focused on the microlevel of distinct gesture types directed towards conspecifics and/or humans (Hopkins et al. 1993; Hopkins et al. 2005b; Fletcher \& Weghorst 2005; Fletcher 2006; Meguerditchian \& Vauclair 2006; Chapelain 2010; Meguerditchian et al. 2012), here we discuss our findings by focusing on four gestures for which we found a right-hand bias at the population level and which were also studied by other authors. The right-hand preference we found for Slap hand at the population level is in accordance with Meguerditchian and Vauclair's (2006) study of olive baboons. Our result showing a right-hand preference at the population level for Extend hand is not in agreement 
with Chapelain's (2010) study of bonobos who found no hand preference for Arm held towards the other (invitation), same gesture as Extend hand but labelled differently. We found a right-hand preference at the population level for Embrace whereas Fletcher and Weghorst's (2005) study of chimpanzees did not. A reason for these contradictory findings might be that these authors considered a global definition of Embrace including not only our Embrace but Embrace lateral, Embrace ventral/dorsal and Embrace half in addition, gestures for which we did not find a right-hand bias at the population level. We found a right-hand preference at the population level for Throw object. This is in accordance with Hopkins and colleagues' (1993, 2005b) studies which first showed in, respectively, a group of 24 and two colonies totalling 89 captive chimpanzees, a right-hand bias at the population level for Throwing directed towards, respectively, humans and towards humans and conspecifics (pooled data). To our knowledge, no information in the literature concerns any of the other nine gestures we studied that presented a right-hand bias at the population level (i.e. Slap foot, Kick, Punch, Hit with object, Attempt to reach, Drag object, Put object on head/back, Shake object and Raise arm). No bias at the population level for Clap hand was detected; however, a majority of subjects (seven of eight) were lateralized. These results agree with the patterns shown by Fletcher (2006) for Clap (an attention-getting behaviour directed towards humans) in a group of 26 captive chimpanzees. Our result differs from Meguerditchian and colleagues' (2012) report showing a predominance of right-hand use for Clapping (same gesture as Clap but labelled differently) in two colonies of captive chimpanzees totalling 94 subjects. Our results for Touch body and Touch genital showing no hand preference at the population level agree with Fletcher and Weghorst's (2005) study of chimpanzees for Touch other and Chapelain's (2010) study of bonobos for Touch body and Touch genital. In addition, we found no hand preference at the population level as Chapelain (2010) did for Embrace lateral and Moving with arms around the partner (same gesture as Embrace half but labelled differently). 
To sum up, our study showing that the majority of the most frequent chimpanzees' intraspecific gestures presented a right-hand bias at the population level overall supports the ELP theory (e.g. Ghirlanda \& Vallortigara 2004) predicting that population level asymmetry should be found in fitness-relevant social behaviours. Our study also supports the Ghirlanda and colleagues' (2009) model postulating that population level biases can be explained by an ESS based on intraspecific interactions. Moreover, these findings support the gestural OHL theory (e.g. Corballis 2002) proposing that gestural laterality represents a precursor of the language left-brain specialization.

Factors and their mutual interactions influencing gestural laterality

We discuss now the selected results of our multifactorial analyses considering modulation of gestural lateralization by emotional processing, communication strategies, social pressures and the demographic factor signaller's age class.

\section{Modulation by emotional processing}

Our findings suggested that signallers' emotional state (emotional valence per se and stress-related emotional states) would affect chimpanzees' gestural laterality through the emotional valence associated with the social interaction (positive versus negative), signallers' hierarchical status and affiliation as well as the position of the signaller in the recipient's visual field during the interaction

Considering emotional valence of the interaction context, we found that signallers' righthand use was more pronounced in negative than in positive contexts when performing common gestures. These findings agree with Rohlfs and Ramirez's (2006) review showing that negative emotional states (e.g. anger), which frequently elicit approach motivation, 
increased activity in humans' left prefrontal brain leading to right-hand preference in negative emotional contexts.

Considering signallers' hierarchical status, subordinate chimpanzees were overall more right-handed than intermediates and dominants. These differences may be the consequence of higher levels of psychosocial stress (e.g. competition for access to food and space) experienced by subordinates leading to a greater right-hand use. Indeed, stress elicits a rightside bias at the population level (rats, Rattus spp.: e.g. Castellano et al. 1989; anoles, Anolis carolinensis: Deckel 1998) possibly because it would inhibit the right hemisphere. This assumption is supported by human studies reporting that stress could induce several neurochemical changes (e.g. increase of dopamine) causing structural and functional alterations in the right hemisphere (see Rohlfs \& Ramirez 2006 for a review). To our knowledge, this is the first evidence of a hierarchical effect on gestural laterality of nonhuman primates.

Considering affiliation, subordinate signallers were less right-handed for gestures towards a strong than towards a medium affiliative subordinate partner. We hypothesize that psychosocial stress effects (that would increase right-hand use as mentioned above) would be less important when subordinates interact with other subordinates and particularly during interactions involving pairs of strong affiliative partners.

Considering the position of the signaller in the recipient's visual field during interaction, our results showed that chimpanzee signallers were overall more right-handed when they were in recipients' left visual field during an interaction (RVF_L) than in recipients' right visual field (RVF_R). We assumed that recipients' more pronounced facial expressions of emotions on the left than on the right hemiface (e.g. chimpanzees: Wallez et al. 2012) could enhance signallers' emotional state during an interaction and would thus explain signallers' 
greater right-hand use in RVF_L. Indeed, as previously detailed, negative emotion and stress are thought to modulate right-hand use.

\section{Modulation by communication strategies}

Our findings suggested that chimpanzees' use of communication strategies depended on gesture characteristics (i.e. tactile, visual or auditory gestures; gestures involving or not the use of a communication tool).

Considering sensory modality, chimpanzees used their right hand to perform tactile gestures (implying physical contact with the recipient) and visual gestures (implying transmission of a visual signal) more when the recipient was in their right visual field during an interaction (SVF_R) than in their left visual field (SVF_L). We hypothesized that they used the hand ipsilateral to the recipient to facilitate transmission of these signals. Conversely to tactile and visual gestures, signallers preferentially used their hand on the side opposite to the recipient (i.e. contralateral hand) for auditory gestures. Personal observations enabled us to hypothesize that when they plan to perform an auditory gesture, they would keep their hand close to the recipient free to be used for further potential tactile or visual gestures towards the recipient (e.g. for a Push).

Considering gestures involving the use of a communication tool, signallers used their right hand more for gestures with an object than for gestures without an object when the recipient was in their left visual field (SVF_L) and conversely in an SVF_R situation. In other words, they preferentially used their hand contralateral to the recipient to communicate with an object. Personal observations suggested that they did so possibly to prevent the recipient from grabbing the potentially coveted object used as a communication tool and/or to keep their hand ipsilateral to the recipient free to be used for a potential additional gesture towards the latter. 


\section{Modulation by social pressures}

According to the ELP theory (e.g. Ghirlanda \& Vallortigara 2004; Vallortigara \& Rogers (2005), the alignment of the direction of laterality at the population level would emerge from social pressures occurring when individually asymmetrical organisms must coordinate their behaviours with those of other asymmetrical organisms of the same or different species. In the present study, we found that the sharing degree of gesture affected chimpanzee signallers' right-hand use. Overall, they used their right hand more for common gestures (i.e. gestures performed by most of the subjects in our population) than for rare gestures (i.e. gestures performed by only a few subjects), possibly because common gestures benefit by being more codified/lateralized than rare gestures, resulting in potentially more coordination that facilitates interactions and thus social cohesion. This facilitation of cohesion would especially benefit chimpanzees living in groups characterized by a higher variable group membership (Aureli et al. 2008). Our study thus provides findings supporting the ELP theory (e.g. Ghirlanda \& Vallortigara 2004) postulating that alignment of laterality would result from social pressures. As far as we know, this is the first evidence of the modulation effect of social pressure on primates' gestural laterality.

We also found a group effect as Leipzig signallers were less right-handed than Beauval signallers for auditory gestures. Subjects in each group are relatively closely related and groups might differ genetically from one another. Laterality of auditory gestures could have been influenced by genetics and/or social learning. Lonsdorf and Hopkins (2005) suggested that their influence could explain variation in laterality patterns of tool use (in noncommunication actions) between groups. Moreover, Taglialatela and colleagues' (2012) study of chimpanzees supports the hypothesis that social learning participates in the acquisition and use of attention-getting vocalizations. This might also be the case in gestural communication as we reported for auditory gestures. 
Note that social pressure effects on gestural laterality have also been found through the influence of signaller's hierarchical status and affiliation.

\section{Modulation by signaller's age class}

The following three age groups emerged from our analysis: immatures and adolescents, young and mature adults, and elders. No difference in right-hand use was found between either immatures and adolescents or young and mature adults. Considering elders, they were less right-handed than adolescent, young and mature adults as well as to a lesser extent than immatures. This decrease in right-hand use by elderly subjects has already been documented in humans (Kalisch et al. 2006). A reason might be that physical limitations and lower activity associated with ageing could decrease the practice-based performance of the right hand that would thus converge towards the performance of the left hand (humans: Hughes et al. 1997; Schut 1998; Ranganathan et al. 2001). We can assume that the lower sociality we observed in elders could also produce the shift towards ambidexterity with ageing in our subjects. To our knowledge, this is the first evidence of a possible senescence effect on manual laterality of nonhuman primates.

Considering the two age groups, immatures-adolescents and young-mature adults, we found an increase in right-hand use with age. This agrees with some reports indicating that right-hand preference for gestures increases with age in both chimpanzees: (Hobaiter \& Byrne 2013; Hopkins \& Leavens 1998) and olive baboons (Meguerditchian \& Vauclair 2006).

To conclude, to our knowledge, our study shows for the first time (1) that individual members of a species (chimpanzees) present a limb bias (right-hand bias) at the population level for several of their most frequent intraspecific communication gestures, (2) that alignment of laterality in gestural communication could result from different types of social 
pressures (i.e. through the influence of signaller's hierarchical status, affiliation, sharing degree of gestures and signaller's group). Our findings thus support (1) the ELP theory (e.g. Ghirlanda \& Vallortigara 2004) proposing that the evolution of population level asymmetries is influenced by fitness-relevant social behaviours and that alignment of laterality would result from social pressures, (2) the Ghirlanda and colleagues' (2009) model predicting that population level biases could be explained by an ESS based on intraspecific interactions and (3) the gestural OHL theory (e.g. Corballis 2002) postulating that laterality in gestural communication represents a precursor of the left-hemispheric lateralization of language. Associated with these findings, we found that some particular gesture characteristics were better markers than others of the right-handedness/left-brain specialization for language (Prieur 2015). By showing complex intertwinement between effects of interaction context, gesture and individual sociodemographic characteristics on gestural laterality, our findings emphasize the need to take into account these effects when investigating social laterality.

To understand better relationships between cerebral lateralization and population level laterality in an evolutionary perspective, it would be especially important to study species varying in their degree of sociality and that researchers agree on a common standardized methodology considering socioecologically relevant contexts (i.e. intraspecific interactions in environments ensuring subjects behave as naturally as possible: in the wild and/or in favourable captive conditions when naturalization of enclosures is stimulating and social groups include many subjects) and multiple potentially influential factors.

\section{Acknowledgments}

We are very grateful to all the keepers, colleagues and researchers of the Wolfgang Köhler Primate Research Center at Zoo Leipzig, Germany, the Zooparc de Beauval and the zoo of La Palmyre, France and particularly Daniel Hanus, Josep Call, Aude Desmoulins, 
Romain Potier, Florence Perroux and Thierry Petit for allowing us to study their chimpanzee groups as well as for their friendliness and helpfulness. We are also indebted to Amandine Chapelain for her advice about the design of the observation protocol and to Maxime Hervé for his statistical advice about the use of 'lme4' and 'lsmeans' packages as well as to Ann Cloarec for correcting the English. This study has been performed in the framework of a Ph.D. funded by the French Ministry of Research and Technology with additional financial support of Rennes Metropole and the VAS Doctoral School. In addition, the study was supported by a Sofja-Kovalevskaja Award of the Alexander von Humboldt Foundation to S.P. (Grant Number DEU/1069105).

\section{References}

Altmann, J. (1974). Observational study of behavior: Sampling methods. Behaviour, 49, 227 267.

Arbib, M. A., Liebal, K., \& Pika, S. (2008). Primate vocalization, gesture, and the evolution of human language. Current Anthropology, 49(6), 1053-1063.

Aureli, F., Schaffner, C. M., Boesch, C., Bearder, S. K., Call, J., Chapman, C. A., ... \& Van Schaik, C. P. (2008). Fission-fusion dynamics. Current Anthropology, 49(4), 627-654.

Baraud, I., Buytet, B., Bec, P., \& Blois-Heulin, C. (2009). Social laterality and 'transversality' in two species of mangabeys: influence of rank and implication for hemispheric specialization. Behavioural brain research, 198(2), 449-458. 
Bates, D., Maechler, M., Bolker, B., \& Walker, S. (2014). lme4: Linear mixed-effects models using Eigen and S4. $R$ package version, 1(7). URL: http://cran.rproject.org/package\%3Dlme4.

Bisazza, A., Cantalupo, C., Capocchiano, M., \& Vallortigara, G. (2000). Population lateralisation and social behaviour: a study with 16 species of fish. Laterality: Asymmetries of Body, Brain and Cognition, 5(3), 269-284.

Bisazza, A., De Santi, A., Bonso, S., \& Sovrano, V. A. (2002). Frogs and toads in front of a mirror: lateralisation of response to social stimuli in tadpoles of five anuran species. Behavioural brain research, 134(1), 417-424.

Bourjade, M., Meunier, H., Blois-Heulin, C., \& Vauclair, J. (2013). Baboons' hand preference for a communicative gesture, but not for a simple manipulative action, is not influenced by spatial factors. Developmental Psychobiology, 55, 651-661.

Call, J. E., \& Tomasello, M. E. (2007). The gestural communication of apes and monkeys. Oxford, U.K: Psychology Press.

Casperd, J. M., \& Dunbar, R. I. M. (1996). Asymmetries in the visual processing of emotional cues during agonistic interactions by gelada baboons. Behavioural Processes, 37, 57-65.

Castellano, M. A., Diaz-Palarea, M. D., Barroso, J., \& Rodriguez, M. (1989). Behavioral lateralization in rats and dopaminergic system: Individual and population laterality. Behavioral Neuroscience, 103(1), 46-53. 
Chapelain, A. (2010). Hand preferences in bonobos (Pan paniscus) for a variety of actions: spontaneous daily actions (non-social and social), bimanual coordination ('tube task'), tool-use ('termite fishing') and induced gestures ('begging'). (doctorate thesis), Loughborough, U.K.: Loughborough University. URL: https://dspace.lboro.ac.uk/dspacejspui/handle/2134/7273.

Chapelain, A., Hogervorst, E., \& Blois-Heulin, C. (n.d.). Laterality in social behaviour in bonobos (Pan paniscus): preliminary results. Manuscript in preparation.

Cochet, H., \& Byrne, R. W. (2013). Evolutionary origins of human handedness: evaluating contrasting hypotheses. Animal Cognition, 16(4), 531-542.

Corballis, M. C. (2002). From hand to mouth: the origins of language. Princeton, NJ: Princeton University Press.

Crockford, C., \& Boesch, C. (2005). Call combinations in wild chimpanzees. Behaviour, 142(4), 397-421.

Deckel, A. W. (1998). Hemispheric control of territorial aggression in Anolis carolinensis: effects of mild stress. Brain, Behavior and Evolution, 51, 33-39.

Fletcher, A. W. (2006). Clapping in chimpanzees: evidence of exclusive hand preference in a spontaneous, bimanual gesture. American Journal of Primatology, 68, 1081-1088. 
Fletcher, A. W., \& Weghorst, J. A. (2005). Laterality of hand function in naturalistically housed chimpanzees (Pan troglodytes). Laterality, 10 (3), 219-242.

Forrester, G. S., Quaresmini, C., Leavens, D. A., Spiezio, C., \& Vallortigara, G. (2012). Target animacy influences chimpanzee handedness. Animal cognition, 15(6), 1121-1127.

Fox, J., \& Weisberg, S. (2011). An \{R\} Companion to Applied Regression, Second Edition. Thousand Oaks, CA: Sage. URL: http://socserv.socsci.mcmaster.ca/jfox/Books/ Companion

Ghirlanda, S., \& Vallortigara, G. (2004). The evolution of brain lateralization: A gametheoretical analysis of population structure. Proceedings of the Royal Society of London, 271(1541), 853-857.

Ghirlanda, S., Frasnelli, E., \& Vallortigara, G. (2009). Intraspecific competition and coordination in the evolution of lateralization. Philosophical Transactions of the Royal Society of London B, 364, 861-866.

Goodall, J. (1986). The chimpanzees of Gombe: Patterns in adaptation. Cambridge, MA: Harvard University Press.

Goodall, J. (1989). Glossary of Chimpanzee Behaviors. Tucson, AZ: Jane Goodall Institute. 
Harris, L. J., \& Carlson, D. F. (1993). Hand preference for visually-guided reaching in human infants and adults. In Primate laterality J. P. Ward \& W. D. Hopkins, Eds (pp. 285-305). New York, NY: Springer.

Harrison, R. M., \& Nystrom, P. (2010). Handedness in captive gorillas (Gorilla gorilla). Primates, 51, 251-261.

Hervé, M. (2014). RVAideMemoire: Diverse basic statistical and graphical functions. R package version 0.9-40. URL: http://CRAN.R-project.org/package=RVAideMemoire.

Hobaiter, C., \& Byrne, R. W. (2013). Laterality in the gestural communication of wild chimpanzees. Annals of the New York Academy of Sciences, 1288(1), 9-16.

Hohmann, G., \& Fruth, B. (2003). Culture in Bonobos? Between-Species and Within-Species Variation in Behavior 1. Current Anthropology, 44(4), 563-571.

Hopkins, W. D., Bard, K. A., Jones, A., \& Bales, S. L. (1993). Chimpanzee hand preference in throwing and infant cradling: implications for the origin of human handedness. Current Anthropology, 786-790.

Hopkins, W. D., \& de Waal, F. B. M. (1995). Behavioral laterality in captive bonobos (Pan paniscus): replication and extension. International Journal of Primatology, 16, 261-276.

Hopkins, W. D., \& Leavens, D. A. (1998). Hand Use and Gestural Communication in Chimpanzees (Pan troglodytes). Journal of Comparative Psychology, 112(1), 95-99. 
Hopkins, W. D., \& Wesley, M. J. (2002). Gestural communication in chimpanzees (Pan troglodytes): The influence of experimenter position on gesture type and hand preference. Laterality, 7(1), 19-30.

Hopkins, W. D., Russell, J. L., Freeman, H., Buehler, N., Reynolds, E., \& Schapiro, S. J. (2005a). The distribution and development of handedness for manual gestures in captive chimpanzees (Pan troglodytes). Psychological Science, 6, 487-493.

Hopkins, W. D., Russell, J. L., Cantalupo, C., Freeman, H.., \& Schapiro, S. J. (2005b). Factors Influencing the Prevalence and Handedness for Throwing in Captive Chimpanzees (Pan troglodytes). Journal of Comparative Psychology, 119 (4), 363-370.

Hopkins, W. D., Pika, S., Liebal, K., Bania, A., Meguerditchian, A., Gardner, M., \& Schapiro, S. (2012). Handedness for manual gestures in great apes: a meta-analysis. In S. Pika, \& K. Liebal (Eds.), Developments in Primate gesture research (pp. 93-110). Amsterdam, Netherlands: John Benjamin's Publishing Company.

Hughes, S., Gibbs, J., Dunlop, D., Edelman, P., Singer, R., \& Chang, R. W. (1997). Predictors of decline in manual performance in older adults. Journal of the American Geriatrics Society, 45(8), 905-910.

Kalisch, T., Wilimzig, C., Kleibel, N., Tegenthoff, M., Dinse, H. R. (2006). Age-Related Attenuation of Dominant Hand Superiority, PLoS One, 1(1), e90. 
Kano, T. (1992). The last ape: Pygmy chimpanzee behavior and ecology. Stanford, CA: Stanford University Press.

Kano, T. (1998). A preliminary glossary of bonobo behaviors atWamba. In T. Nishida (Ed.), Comparative study of the behavior of the genus Pan by compiling video ethogram (pp. 3981). Kyoto, Japan: Nissho Printer.

Kimura, D. (1973). Manual activity during speaking- I. Right-handers. Neuropsychologia, $11(1), 45-50$.

Langbein, J., \& Puppe, B. (2004). Analysing dominance relationships by sociometric methods - a plea for a more standardised and precise approach in farm animals. Applied Animal Behaviour Science, 87, 293-315.

Lenth, R. V. (2014). 1smeans: Least-Squares Means. R package version 2.11. URL: http://CRAN.R-project.org/package=lsmeans.

Lonsdorf, E. V., \& Hopkins, W. D. (2005). Wild chimpanzees show population level handedness for tool use. Proceedings of the National Academy of Sciences of the United States of America, 102, 12634-12638.

Marchant, L. F., \& McGrew, W. C. (1991). Laterality of function in apes: a meta-analysis of methods. Journal of Human Evolution, 21, 425-438. 
McGrew, W. C., \& Marchant, L. F. (1997). On the other hand: current issues in and metaanalysis of the behavioral laterality of hand function in nonhuman primates. Yearbook of Physical Anthropology, 40, 201-232.

McNeill, D. (1992). Hand and Mind: What Gestures Reveal About Thought. Chicago, IL: University of Chicago Press.

McNeill, D. (2012). How Language Began, Gesture and Speech in Human Evolution. Cambridge, U.K.: Cambridge University Press.

Meguerditchian, A., \& Vauclair, J. (2006). Baboons communicate with their right hand. Behavioural Brain Research, 171(1), 170-174.

Meguerditchian, A., Vauclair, J., \& Hopkins, W. D. (2010). Captive chimpanzees use their right hand to communicate with each other: Implications for the origin of the cerebral substrate for language. Cortex, 46(1), 40-48.

Meguerditchian, A., Molesti, S., \& Vauclair, J. (2011). Right-handedness predominance in 162 baboons (Papio anubis) for gestural communication: Consistency across time and groups. Behavioral Neuroscience, 125(4), 653.

Meguerditchian, A., Gardner, M. J., Schapiro, S. J., \& Hopkins, W. D. (2012). The sound of one hand clapping: Handedness and perisylvian neural correlates of a communicative gesture in chimpanzees. Proceeding of the Royal Society B: Biological Science, 279, 19591966. 
Meguerditchian, A., Vauclair, J., \& Hopkins, W. D. (2013). On the origins of human handedness and language: A comparative review of hand preferences for bimanual coordinated actions and gestural communication in nonhuman primates. Developmental Psychobiology, 55, 637-650.

Nishida, T., Kano, T., Goodall, J., McGrew, W. C., \& Nakamura, M. (1999). Ethogram and Ethnography of Mahale Chimpanzees. Anthropological Science, 107(2), 141-188.

Nishida, T., Zamma, K., Matsusaka, T., Inaba, A., \& McGrew, W. C. (Eds.) (2010). Chimpanzee behavior in the wild: an audio-visual encyclopedia. Tokyo, Japan: Springer.

Parr, L. A., \& Waller, B. M. (2006). Understanding chimpanzee facial expression: Insights into the evolution of communication. Social Cognitive and Affective Neuroscience, 1, 221228.

Pika, S., Liebal, K., \& Tomasello, M. (2003). Gestural communication in young gorillas (Gorilla gorilla): gestural repertoire, learning, and use. American Journal of Primatology, $60,95-111$.

Pika, S., Liebal, K., \& Tomasello, M. (2005). Gestural communication in subadult bonobos (Pan paniscus): repertoire and use. American Journal of Primatology, 65, 39-61.

Pika, S., \& Bugnyar, T. (2011). The use of referential gestures in ravens (Corvus corax) in the wild. Nature Communications, 2, 560. 
Plooij, F. X. (1984). The behavioral development of free-living chimpanzee babies and infants. Norwood, NJ: Ablex Publishing Corporation.

Pollick, A. S., \& de Waal, F. (2007). Ape gestures and language evolution. Proceedings of the National Academy of Sciences of the United States of America, 104, 8184-8189.

Prieur, J. (2015). Chimpanzees' and gorillas' intraspecific gestural laterality: a multifactorial investigation. (doctorate thesis), Rennes, France: University of Rennes 1. URL: http://www.theses.fr/en/2015REN1S056.

R Development Core Team. (2014). R: A language and environment for statistical computing. Vienna, Austria: R Foundation for Statistical Computing. URL: http://http//www.Rproject.org.

Ranganathan, V. K., Siemionow, V., Sahgal, V., Yue, G. H. (2001). Effects of Aging on Hand Function, Journal of the American Geriatrics Society, 49(11), 1478-1484.

Rogers, L. J. (2000). Evolution of hemispheric specialization: advantages and disadvantages. Brain and Language, 73, 236-253.

Rogers, L. J., Zucca P., \& Vallortigara, G. (2004). Advantages of having a lateralized brain. Proceedings of the Royal Society B, 271, S420-S422.

Rohlfs, P., \& Ramirez, J. M. (2006). Aggression and brain asymmetries: a theoretical overview. Aggression and Violent Behavior, 11, 283-297. 
Roth, R. R. (1995). A study of gestural communication during sexual behaviour in Bonobos (Pan paniscus Schwartz). Calgary, Canada: University of Calgary Press.

Schut, L. J. (1998). Motor system changes in the aging brain: what is normal and what is not. Geriatrics, 53 (1) S16-9.

Siegel, S., \& Castellan, N.J. (1988). Nonparametric statistics for the behavioural sciences. Singapore: McGraw-Hill.

Silk, J., Cheney, D., \& Seyfarth, R. (2013). A practical guide to the study of social relationships. Evolutionary Anthropology, 22, 213-225.

Taglialatela, J. P., Reamer, L., Schapiro, S. J., \& Hopkins, W. D. (2012). Social learning of a communicative signal in captive chimpanzees. Biology Letters, 8(4), 498-501.

Tomasello, M., Gust, D., \& Frost, G. T. (1989). A longitudinal investigation of gestural communication in young chimpanzees. Primates, 30, 35-50.

Vallortigara, G., \& Rogers, L. J. (2005). Survival with an asymmetrical brain: advantages and disadvantages of cerebral lateralization. Behavioral and Brain Sciences, 28, 575-589.

Van Hooff, J. A. R. A. M. (1973). A structural analysis of the social behaviour of a semicaptive group of chimpanzees. In M. Von Cranach \& I. Vine (Eds.). Social Communication and Movement. 75-162). London, U.K.: European Association of Experimental Social Psychology. 
de Vries, H. (1995). An improved test of linearity in dominance hierarchies containing unknown or tied relationships. Animal Behaviour, 50, 1375-1389.

de Vries, H. (1998). Finding a dominance order most consistent with a linear hierarchy: a new procedure and review. Animal Behaviour, 55, 827-843.

de Vries, H., Stevens, J. M. G., \& Vervaecke, H. (2006). Measuring and testing the steepness of dominance hierarchies. Animal Behaviour, 71, 585-592.

De Waal, F. B. (1988). The communicative repertoire of captive bonobos (Pan paniscus), compared to that of chimpanzees. Behaviour, 106(3), 183-251.

Waller, B. M., Warmelink, L., Liebal, K., Micheletta, J. \& Slocombe, K. E. (2013). Pseudoreplication: a widespread problem in primate communication research. Animal Behaviour, 86(2), 483-488.

Wallez, C., Schaeffer, J. A., Meguerditchian, A., Vauclair, J., Schapiro, S. J., \& Hopkins, W. D. (2012). Contrast of hemispheric lateralization for oro-facial movements between learned attention-getting sounds and species-typical vocalizations in chimpanzees: Extension in a second colony. Brain and Language, 123, 75-79.

Weaver, A., \& de Waal, F. B. M. (2002). An index of relationship quality based on attachment theory. Journal of Comparative Psychology, 116, 93-106. 


\begin{tabular}{|c|c|c|}
\hline Gesture & Description & Source(s) \\
\hline Clap hand * & One open hand (more often the one in the upper position) strikes against the other hand & Call \& Tomasello (2007) \\
\hline Slap foot $*$ & Subject hits ground/wall/object with the sole or heel of one foot & Pika et al. $(2003,2005)$ \\
\hline Slap hand * & Subject hits ground/wall/object with the palm of one hand & Pika et al. $(2003,2005)$ \\
\hline Embrace & One arm of signaller is stretched and raised up to about head level with palm facing downwards or placed lightly on the recipient's body & Roth (1995) \\
\hline Embrace half & Subject puts one arm around another subject while walking & Nishida et al. $(1999,2010)$ \\
\hline Embrace lateral * & $\begin{array}{l}\text { Subject places one arm gently around the other's shoulder, back, or waist, or puts both arms around the other while pulling the recipient closer; both } \\
\text { partners are initially side by side and facing the same direction }\end{array}$ & de Waal (1988) \\
\hline Embrace ventral/dorsal * & Both arms are opened and the partner is hugged ventro/dorsoventrally (leading arm recorded), with belly contact & de Waal (1988) \\
\hline Hand on & The palm of one hand is placed on the head of another subject and stays there $>2 \mathrm{~s}$ & Pika et al. $(2003,2005)$ \\
\hline Hit with object* & Subject clubs another subject with object (e.g. branch) held in one hand & Nishida et al. $(1999,2010)$ \\
\hline Kick * & $\begin{array}{l}\text { Any sort of contact made with the sole/heel or fingers of one foot with another subject, without appreciable force, but the actual contact is more forceful } \\
\text { than a simple laying of foot on another's body }\end{array}$ & Pollick \& de Waal (2007) \\
\hline Punch * & $\begin{array}{l}\text { Any sort of contact made with fist/wrist or fingers of one hand with another subject, without appreciable force, but the actual contact is more forceful } \\
\text { than a simple laying of the hand on another's body }\end{array}$ & Pollick \& de Waal (2007) \\
\hline Push & Gentle pressure applied against another subject with one hand or arm & Call \& Tomasello (2007) \\
\hline Touch body $*$ & Gentle and brief $(<5 \mathrm{~s})$ contact of the recipient's body (except genitals) with one hand or arm & Pika et al. $(2003,2005)$ \\
\hline Touch genital * & Gentle and brief $(<5 \mathrm{~s})$ contact of the recipient's genitals with the flat of one hand & Pika et al. $(2003,2005)$ \\
\hline Attempt to reach * & Subject briefly extends hand (with fingers slightly flexed with palm up or down) towards another subject, as an attempt to touch/catch it & Pika et al. $(2003,2005)$ \\
\hline Drag object & Subject pulls an object (e.g. branch) on the ground with one hand towards another subject & Nishida et al. $(1999,2010)$ \\
\hline Extend hand * & Subject outstretches one hand or arm (wrist and/or fingers extended with palm up or down) towards another subject; hand or arm remains stationary & Goodall (1989) \\
\hline Put object on head/back * & Subject places an object (e.g. branch) on its head/back with one hand & Nishida et al. (2010) \\
\hline Raise arm & Subject lifts one out-stretched arm (all or only forearm) overhead in a quick jerky movement with fingers slightly flexed & Plooij (1984) \\
\hline Shake object * & An object (e.g. branch) is moved back and forth with quick jerky movements of one arm, slightly or vigorously, while the subject is sitting or standing & Kano $(1992,1998)$ \\
\hline Throw object $*$ & Subject sends an object (e.g. branch) through the air with one hand towards another subject & Hohmann \& Fruth (2003) \\
\hline
\end{tabular}

Gestures are grouped by sensory modality (three auditory, 11 tactile and seven visual gestures) and presented in alphabetical order. Gestures marked with * are followed by descriptions inspired by the mentioned source(s), except for Extend hand, they are labelled differently because details based on personal observations have been added. 
Table 2. Characteristics, descriptive statistics and analyses of each gesture

\begin{tabular}{|c|c|c|c|c|c|c|c|c|c|c|c|c|c|c|c|}
\hline Gesture & $\begin{array}{l}\text { Sensory } \\
\text { modality }\end{array}$ & $\begin{array}{c}\text { Communication } \\
\text { tool }\end{array}$ & Duration & $\begin{array}{l}\text { Sharing } \\
\text { degree }\end{array}$ & $N$ & $\begin{array}{c}\text { Data points } \\
\text { analysed }\end{array}$ & Non-lat. & $\begin{array}{l}\text { B test Lat. } \\
\text { vs. Non-lat. }\end{array}$ & LH & $\mathrm{RH}$ & $\begin{array}{l}\text { B test LH } \\
\text { vs. RH }\end{array}$ & $\begin{array}{c}\text { Mean } \\
\text { HI }\end{array}$ & Shapiro test & t-test/Wilcoxon test & $\begin{array}{l}\text { Mean } \\
\text { ABSHI }\end{array}$ \\
\hline Clap hand & Auditory & - & Short & Rare & 8 & 177 & 1 & 0.070 & 4 & 3 & 1 & -0.151 & 0.009 & $\mathrm{~W}=16.5, P=0.889$ & 0.836 \\
\hline Slap hand & Auditory & - & Short & Common & 33 & 2850 & 16 & 1 & 0 & 17 & $\mathbf{0}$ & 0.391 & 0.867 & $\mathrm{t}=0.391, P<\mathbf{0 . 0 0 0 1}$ & 0.400 \\
\hline Slap foot & Auditory & - & Short & Common & 21 & 1412 & 10 & 1 & 0 & 11 & 0.001 & 0.468 & 0.012 & $\mathrm{~W}=223.5, P=\mathbf{0 . 0 0 0 2}$ & 0.513 \\
\hline Touch genital & Tactile & - & Long & Common & 29 & 692 & 25 & 0.0001 & 2 & 2 & - & -0.079 & 0.299 & $\mathrm{t}=-0.079, P=0.237$ & 0.261 \\
\hline Hand on & Tactile & - & Long & Common & 30 & 581 & 23 & 0.005 & 5 & 2 & 0.453 & -0.052 & 0.474 & $\mathrm{t}=-0.052, P=0.472$ & 0.281 \\
\hline Embrace lateral & Tactile & - & Long & Common & 29 & 1339 & 25 & 0.0001 & 2 & 2 & - & 0.016 & 0.044 & $\mathrm{~W}=219, P=0.478$ & 0.236 \\
\hline Embrace ventral/dorsal & Tactile & - & Long & Rare & 13 & 686 & 10 & 0.092 & 1 & 2 & - & 0.056 & 0.925 & $\mathrm{t}=0.077, P=0.107$ & 0.224 \\
\hline Touch body & Tactile & - & Long & Common & 39 & 4203 & 35 & $\mathbf{0}$ & 1 & 3 & - & 0.060 & 0.011 & $\mathrm{~W}=456.5, P=0.215$ & 0.149 \\
\hline Embrace half & Tactile & - & Long & Rare & 12 & 623 & 11 & 0.006 & 0 & 1 & - & 0.064 & 0.353 & $\mathrm{t}=0.064, P=0.264$ & 0.154 \\
\hline Push & Tactile & - & Short & Common & 24 & 464 & 20 & 0.002 & 0 & 4 & - & 0.101 & 0.618 & $\mathrm{t}=0.101, P=0.113$ & 0.260 \\
\hline Embrace & Tactile & - & Long & Common & 31 & 771 & 28 & $\mathbf{0}$ & 1 & 2 & - & 0.188 & 0.759 & $\mathrm{t}=0.188, P=\mathbf{0 . 0 0 0 8}$ & 0.276 \\
\hline Kick & Tactile & - & Short & Rare & 8 & 95 & 8 & 0.008 & 0 & 0 & - & 0.291 & 0.558 & $\mathrm{t}=0.291, P=\mathbf{0 . 0 0 9}$ & 0.291 \\
\hline Punch & Tactile & - & Short & Common & 34 & 1654 & 18 & 0.864 & 0 & 16 & $\mathbf{0}$ & 0.317 & 0.858 & $\mathrm{t}=0.317, P<\mathbf{0 . 0 0 0 1}$ & 0.348 \\
\hline Hit with object & Tactile & Yes & Short & Rare & 12 & 248 & 7 & 0.774 & 0 & 5 & - & 0.466 & 0.745 & $\mathrm{t}=0.466, P=\mathbf{0 . 0 0 0 4}$ & 0.491 \\
\hline Attempt to reach & Visual & - & Short & Common & 31 & 831 & 23 & 0.011 & 1 & 7 & 0.070 & 0.202 & 0.973 & $\mathrm{t}=0.202, P=\mathbf{0 . 0 0 3}$ & 0.325 \\
\hline Drag object & Visual & Yes & Long & Rare & 13 & 488 & 11 & 0.023 & 0 & 2 & - & 0.257 & 0.845 & $\mathrm{t}=0.256, P=\mathbf{0 . 0 0 0 5}$ & 0.282 \\
\hline Put object on head/back & Visual & Yes & Short & Rare & 11 & 386 & 6 & 1 & 0 & 5 & - & 0.302 & 0.591 & $\mathrm{t}=0.302, P=\mathbf{0 . 0 2 4}$ & 0.398 \\
\hline Shake object & Visual & Yes & Short & Common & 38 & 5095 & 18 & 0.871 & 1 & 19 & $\mathbf{0}$ & 0.314 & 0.340 & $\mathrm{t}=0.314, P<\mathbf{0 . 0 0 0 1}$ & 0.352 \\
\hline Extend hand & Visual & - & Long & Common & 37 & 1226 & 21 & 0.511 & 0 & 16 & $\mathbf{0}$ & 0.381 & 0.860 & $\mathrm{t}=0.381, P<\mathbf{0 . 0 0 0 1}$ & 0.394 \\
\hline Throw object & Visual & Yes & Short & Rare & 12 & 347 & 5 & 0.774 & 1 & 6 & 0.125 & 0.411 & 0.056 & $\mathrm{t}=0.411, P=\mathbf{0 . 0 2 1}$ & 0.598 \\
\hline Raise arm & Visual & - & Short & Common & 25 & 856 & 11 & 0.690 & 0 & 14 & 0.0001 & 0.471 & 0.017 & $\mathrm{~W}=311, P<\mathbf{0 . 0 0 0 1}$ & 0.543 \\
\hline
\end{tabular}

Gestures are regrouped by sensory modality and classified by increasing HI values. $N$ : number of subjects who performed at least 6 times each gesture; Data points analysed: number of data points associated with the $N$ analysed subjects; Non-lat.: numbers of non-lateralized subjects; B test Lat. vs. Non-lat.: p-value of the binomial test on the numbers of lateralized versus nonlateralized subjects; LH: number of left-handed subjects; RH: number of right-handed subjects; B test LH vs. RH: p-value of the binomial test on the numbers of left-handed versus right-handed subjects; -: insufficient number of lateralized subjects for testing; Mean HI: Mean Handedness Index score of $N$ analysed subjects, the sign indicates the direction of the gestural bias (negative value: left-hand bias, positive value: right-hand bias); t-test: t-value and p-value of the t-test only performed for normally distributed HI values of $N$ analysed subjects; Wilcoxon test: W-value and p-value of the Wilcoxon test only performed when normality of HI values is not verified; Mean ABSHI: Mean Absolute value of Handedness Index score of $N$ analysed subjects. Significant results are in bold. 
Table 3. Generalized linear mixed model with dependent, fixed and random variables, their type and associated levels

\begin{tabular}{|c|c|}
\hline Name & Type \\
\hline \multicolumn{2}{|l|}{ Dependent variable } \\
\hline Hand use & Dichotomous (L/R) \\
\hline \multicolumn{2}{|l|}{ Fixed variables } \\
\hline \multicolumn{2}{|l|}{ Individual characteristics (ELP theory) } \\
\hline Position of recipient in signaller's visual field during interaction (SVF) & Dichotomous (L/R) \\
\hline Position of signaller in recipient's visual field during interaction (RVF) & Dichotomous (L/R) \\
\hline Emotional context of interaction & Dichotomous (Negative/Positive) \\
\hline Signaller's sex & Dichotomous (F/M) \\
\hline Signaller's age class & Ordinal (Immature/Adolescent/Young adult/Mature adult/Elder) \\
\hline Recipient's sex & Dichotomous (F/M) \\
\hline Recipient's age class & Ordinal (Immature/Adolescent/Young adult/Mature adult/Elder) \\
\hline Zoo & Nominal (Beauval/Leipzig/Palmyre) \\
\hline Signaller's hierarchical rank & Ordinal (Dominant/Intermediate/Subordinate) \\
\hline Recipient's hierarchical rank & Ordinal (Dominant/Intermediate/Subordinate) \\
\hline Kinship & Nominal (Parent-infant/Siblings/Unrelated) \\
\hline Affiliation & Ordinal (Low/Medium/Strong) \\
\hline \multicolumn{2}{|l|}{ Gesture characteristics (OHL theory) } \\
\hline Sensory modality & Nominal (Auditory/Tactile/Visual) \\
\hline Communication tool & Dichotomous (Yes/No) \\
\hline Duration & Dichotomous (Short/Long) \\
\hline Sharing degree & Dichotomous (Rare/Common) \\
\hline \multicolumn{2}{|l|}{ Random variables } \\
\hline Signaller's identity & Nominal \\
\hline Recipient's identity & Nominal \\
\hline
\end{tabular}

L: Left; R: Right; F: Female; M: Male. 
Table 4. Generalized linear mixed model: summary of the selected results; influence of interactional context

\begin{tabular}{|c|c|c|c|c|c|c|c|}
\hline \multirow{2}{*}{ Fixed variables } & & \multicolumn{2}{|c|}{$\begin{array}{l}\text { Position of recipient in signaller's } \\
\text { visual field during interaction (SVF) }\end{array}$} & \multicolumn{2}{|c|}{$\begin{array}{l}\text { Position of signaller in recipient's } \\
\text { visual field during interaction (RVF) }\end{array}$} & \multicolumn{2}{|c|}{$\begin{array}{c}\text { Emotional } \\
\text { context }\end{array}$} \\
\hline & & SVF_R >SFV_L & SVF_R<SFV_L & RVF_L $>$ RFV_R & RVF_L<RFV_R & $\mathrm{N}>\mathrm{P}$ & $\mathrm{N}<\mathrm{P}$ \\
\hline $\begin{array}{l}\text { Position of recipient in signaller's visual field } \\
\text { during interaction (SVF) }\end{array}$ & $\begin{array}{l}\text { SVF_R } \\
\text { SVF_L }\end{array}$ & & & & & & \\
\hline $\begin{array}{l}\text { Position of signaller in recipient's visual field } \\
\text { during interaction (RVF) }\end{array}$ & $\begin{array}{l}\text { RVF_R } \\
\text { RVF_L }\end{array}$ & & & & & & \\
\hline Emotional context & $\begin{array}{l}\text { Positive }(\mathrm{P}) \\
\text { Negative }(\mathrm{N})\end{array}$ & & & & & & \\
\hline Gestures & $\begin{array}{l}\text { Tactile (T) } \\
\text { Visual (V) } \\
\text { Auditory (A) } \\
\text { With object } \\
\text { Without object } \\
\text { Short } \\
\text { Long } \\
\text { Rare (R) } \\
\text { Common (C) }\end{array}$ & $\begin{array}{l}\mathrm{x} \\
\mathrm{x} \\
\mathrm{x}\end{array}$ & $\mathrm{x}$ & $\begin{array}{l}\mathrm{x} \\
\mathrm{x} \\
\mathrm{x} \\
\mathrm{x} \\
\mathrm{x} \\
\mathrm{x}\end{array}$ & & . & \\
\hline Kinship & $\begin{array}{l}\text { Parent-infant } \\
\text { Siblings } \\
\text { Unrelated }\end{array}$ & & & $\begin{array}{l}\mathrm{x} \\
\mathrm{x}\end{array}$ & & & \\
\hline Signaller's hierarchical rank & $\begin{array}{l}\text { Subordinate (Sub) } \\
\text { Intermediate (Int) } \\
\text { Dominant (Dom) }\end{array}$ & & & $\begin{array}{l}\mathrm{x} \\
\mathrm{x} \\
\mathrm{x}\end{array}$ & & & \\
\hline Affiliation & $\begin{array}{l}\text { Strong (St) } \\
\text { Medium (M) } \\
\text { Low }\end{array}$ & & & & & & \\
\hline Signaller's age class & $\begin{array}{l}\text { Immature (Im) } \\
\text { Adolescent (Ad) } \\
\text { Young Adult (YA) }\end{array}$ & & & $\begin{array}{l}\mathrm{x} \\
\mathrm{x} \\
\mathrm{x}\end{array}$ & & & \\
\hline Zoo & $\begin{array}{l}\text { La Palmyre } \\
\text { Beauval (B) } \\
\text { Leipzig (Le) }\end{array}$ & & & $\begin{array}{l}\mathrm{x} \\
\mathrm{x} \\
\mathrm{x}\end{array}$ & & & \\
\hline
\end{tabular}

L: Left; R: Right; A>B: means 'signallers used their right hand more when A than when B'; X: statistical evidence. 
Table 5. Generalized linear mixed model: summary of the selected results; influence of gesture characteristics

\begin{tabular}{|c|c|c|c|c|c|}
\hline \multirow{2}{*}{ Fixed variables } & & \multicolumn{2}{|c|}{ Communication tool } & \multicolumn{2}{|c|}{ Sharing degree } \\
\hline & & Without $>$ With object & Without $<$ With object & $C>R$ & $\mathrm{C}<\mathrm{R}$ \\
\hline $\begin{array}{l}\text { Position of recipient in signaller's visual field } \\
\text { during interaction }(\mathrm{SVF})\end{array}$ & $\begin{array}{l}\text { SVF_R } \\
\text { SVF_L }\end{array}$ & $\mathrm{x}$ & $\mathrm{x}$ & & \\
\hline $\begin{array}{l}\text { Position of signaller in recipient's visual field } \\
\text { during interaction (RVF) }\end{array}$ & $\begin{array}{l}\text { RVF_R } \\
\text { RVF_L }\end{array}$ & & & & \\
\hline Emotional context & $\begin{array}{l}\text { Positive }(\mathrm{P}) \\
\text { Negative }(\mathrm{N})\end{array}$ & & & $\mathrm{x}$ & \\
\hline Gestures & $\begin{array}{l}\text { Tactile (T) } \\
\text { Visual (V) } \\
\text { Auditory (A) } \\
\text { With object } \\
\text { Without object } \\
\text { Short } \\
\text { Long } \\
\text { Rare (R) } \\
\text { Common (C) }\end{array}$ & & & $\mathrm{x}$ & $\mathrm{x}$ \\
\hline Kinship & $\begin{array}{l}\text { Parent-infant } \\
\text { Siblings } \\
\text { Unrelated }\end{array}$ & & & & \\
\hline Signaller's hierarchical rank & $\begin{array}{l}\text { Subordinate (Sub) } \\
\text { Intermediate (Int) } \\
\text { Dominant (Dom) }\end{array}$ & & & $\mathrm{x}$ & \\
\hline Affiliation & $\begin{array}{l}\text { Strong (St) } \\
\text { Medium (M) } \\
\text { Low }\end{array}$ & & & $\mathrm{x}$ & \\
\hline Signaller's age class & $\begin{array}{l}\text { Immature (Im) } \\
\text { Adolescent (Ad) } \\
\text { Young Adult (YA) }\end{array}$ & & & $\mathrm{x}$ & \\
\hline Zoo & $\begin{array}{l}\text { La Palmyre } \\
\text { Beauval (B) } \\
\text { Leipzig (Le) }\end{array}$ & & & $\mathrm{x}$ & \\
\hline
\end{tabular}

L: Left; R: Right; A>B: means 'signallers used their right hand more when A than when B'; X: statistical evidence. 
Table 6. Generalized linear mixed model: summary of the selected results; influence of individual social characteristics

\begin{tabular}{|c|c|c|c|c|}
\hline \multirow{2}{*}{ Fixed variables } & & \multicolumn{2}{|c|}{ Signaller's hierarchical rank } & \multirow{2}{*}{$\begin{array}{c}\text { Affiliation } \\
\mathrm{M}>\mathrm{St}\end{array}$} \\
\hline & & Sub>Int & Sub>Dom & \\
\hline \multirow{2}{*}{$\begin{array}{l}\text { Position of recipient in signaller's visual field during } \\
\text { interaction (SVF) }\end{array}$} & SVF_R & & $\mathrm{x}$ & \\
\hline & SVF_L & $\mathrm{x}$ & & \\
\hline \multirow{2}{*}{$\begin{array}{l}\text { Position of signaller in recipient's visual field } \\
\text { during interaction (RVF) }\end{array}$} & RVF_R & & & \\
\hline & RVF_L & $\mathrm{x}$ & & \\
\hline \multirow[t]{2}{*}{ Emotional context } & Positive $(\mathrm{P})$ & & & \\
\hline & Negative (N) & & & \\
\hline \multirow[t]{9}{*}{ Gestures } & Tactile $(\mathrm{T})$ & $\mathrm{x}$ & & \\
\hline & Visual (V) & & & \\
\hline & Auditory (A) & & & \\
\hline & With object & & & \\
\hline & Without object & & & \\
\hline & Short & & & \\
\hline & Long & & & \\
\hline & Rare (R) & $\mathrm{x}$ & $\mathrm{x}$ & \\
\hline & Common $(\mathrm{C})$ & & & \\
\hline \multirow[t]{3}{*}{ Kinship } & Parent-infant & & & \\
\hline & Siblings & & & \\
\hline & Unrelated & & & \\
\hline \multirow[t]{3}{*}{ Signaller's hierarchical rank } & Subordinate (Sub) & & & $\mathrm{x}$ \\
\hline & Intermediate (Int) & & & \\
\hline & Dominant (Dom) & & & \\
\hline \multirow[t]{3}{*}{ Affiliation } & Strong $(\mathrm{St})$ & & & \\
\hline & Medium (M) & $\mathrm{x}$ & $\mathrm{x}$ & \\
\hline & Low & & & \\
\hline \multirow[t]{3}{*}{ Signaller's age class } & Immature (Im) & & & \\
\hline & Adolescent (Ad) & & & \\
\hline & Young Adult (YA) & & & \\
\hline \multirow[t]{3}{*}{ Zoo } & La Palmyre & & & \\
\hline & Beauval (B) & & & \\
\hline & Leipzig (Le) & & & \\
\hline
\end{tabular}

L: Left; R: Right; A>B: means 'signallers used their right hand more when A than when B'; X: statistical evidence. 
Table 7. Generalized linear mixed model: summary of the selected results; influence of individual demographic characteristics

\begin{tabular}{|c|c|c|c|c|c|c|c|c|}
\hline \multirow{2}{*}{ Fixed variables } & & \multicolumn{6}{|c|}{ Signaller's age class } & \multirow{2}{*}{$\begin{array}{l}\text { Signaller's group (zoo) } \\
\qquad \text { B }>\text { Le }\end{array}$} \\
\hline & & $\mathrm{E}<\mathrm{MA}$ & $\mathrm{E}<\mathrm{YA}$ & $\mathrm{E}<\mathrm{Ad}$ & $\mathrm{E}<\mathrm{Im}$ & $\mathrm{MA}>\mathrm{Ad}$ & $\mathrm{MA}>\mathrm{Im}$ & \\
\hline $\begin{array}{l}\text { Position of recipient in signaller's visual field } \\
\text { during interaction (SVF) }\end{array}$ & $\begin{array}{l}\text { SVF_R } \\
\text { SVF_L }\end{array}$ & & & & & & & \\
\hline \multirow{2}{*}{$\begin{array}{l}\text { Position of signaller in recipient's visual field } \\
\text { during interaction (RVF) }\end{array}$} & RVF_R & $\mathrm{x}$ & $\mathrm{x}$ & $\mathrm{x}$ & & $\mathrm{x}$ & $\mathrm{x}$ & \\
\hline & RVF_L & $\mathrm{x}$ & $\mathrm{x}$ & $\mathrm{x}$ & & & $\mathrm{x}$ & \\
\hline Emotional context & $\begin{array}{l}\text { Positive }(\mathrm{P}) \\
\text { Negative }(\mathrm{N})\end{array}$ & & & & & & & \\
\hline \multirow[t]{9}{*}{ Gestures } & Tactile (T) & $\mathrm{x}$ & $\mathrm{x}$ & $\mathrm{x}$ & & & $\mathrm{x}$ & \\
\hline & Visual (V) & $\mathrm{x}$ & & & & & & \\
\hline & Auditory (A) & $\mathrm{x}$ & & $\mathrm{x}$ & & & & $\mathrm{x}$ \\
\hline & With object & $\mathrm{x}$ & $\mathrm{x}$ & $\mathrm{x}$ & $\mathrm{x}$ & & $\mathrm{x}$ & \\
\hline & Without object & $\mathrm{x}$ & & & & & $\mathrm{x}$ & \\
\hline & Short & & & & & & & \\
\hline & Long & & & & & & & \\
\hline & Rare (R) & $\mathrm{x}$ & $\mathrm{x}$ & $\mathrm{x}$ & & $\mathrm{x}$ & $\mathrm{x}$ & \\
\hline & Common $(\mathrm{C})$ & & $\mathrm{x}$ & $\mathrm{x}$ & & & & \\
\hline Kinship & $\begin{array}{l}\text { Parent-infant } \\
\text { Siblings } \\
\text { Unrelated }\end{array}$ & & & & & & & \\
\hline Signaller's hierarchical rank & $\begin{array}{l}\text { Subordinate (Sub) } \\
\text { Intermediate (Int) } \\
\text { Dominant (Dom) }\end{array}$ & & & & & & & \\
\hline \multirow[t]{3}{*}{ Affiliation } & Strong (St) & $\mathrm{x}$ & & $\mathrm{x}$ & & & & \\
\hline & Medium (M) & $\mathrm{x}$ & $\mathrm{x}$ & $\mathrm{x}$ & & & $\mathrm{x}$ & \\
\hline & Low & $\mathrm{x}$ & $\mathrm{x}$ & & & & $\mathrm{x}$ & \\
\hline Signaller's age class & $\begin{array}{l}\text { Immature (Im) } \\
\text { Adolescent (Ad) } \\
\text { Young Adult (YA) }\end{array}$ & & & & & & & \\
\hline Zoo & $\begin{array}{l}\text { La Palmyre } \\
\text { Beauval (B) } \\
\text { Leipzig (Le) }\end{array}$ & & & & & & & \\
\hline
\end{tabular}

L: Left; R: Right; MA: Mature Adult; E: Elder; A>B: means 'signallers used their right hand more when A than when B'; X: statistical evidence. 